\title{
Triangle-free subgraphs with large fractional chromatic number
}

\author{
Bojan Mohar* \\ Department of Mathematics \\ Simon Fraser University \\ Burnaby, BC, Canada \\ mohar@sfu.ca
}

\author{
Hehui $\mathrm{Wu}^{\dagger}$ \\ Shanghai Center for Math. Sci. \\ Fudan University \\ Shanghai, China \\ hhwu@fudan.edu.cn
}

August 7, 2018

\begin{abstract}
It is well known that for any integers $k$ and $g$, there is a graph with chromatic number at least $k$ and girth at least $g$. In 1960's, Erdös and Hajnal conjectured that for any $k$ and $g$, there exists a number $h(k, g)$, such that every graph with chromatic number at least $h(k, g)$ contains a subgraph with chromatic number at least $k$ and girth at least $g$. In 1977, Rödl proved the case for $g=4$ and arbitrary $k$. We prove the fractional chromatic number version of Rödl's result.
\end{abstract}

\section{Outline}

A well known result, proved by Erdös in 1950s [6], tells us that for every $k$ and $g$, there exists a graph with chromatic number at least $k$ and girth at least $g$. In 1960s, Erdős and Hajnal proposed the following [7].

Conjecture 1 (Erdős and Hajnal (1969)). For every positive integers $k$ and $g$, there exists an integer $h(k, g)$ such that every graph $G$ with $\chi(G) \geq h(k, g)$ contains a subgraph with chromatic number at least $k$ and girth at least $g$.

In 1977, Rödl [13] proved the conjecture for $g=4$ and arbitrary $k$. The special case when $g=4$ speaks about triangle-free subgraphs. This is the only nontrivial case for which the Erdös-Hajnal conjecture has been confirmed.

Theorem 2 (Rödl (1977)). For every positive integer $k$, there exists an integer $f(k)$ such that if $\chi(G) \geq f(k)$ then $G$ contains a triangle-free subgraph $H$ with $\chi(H)=k$.

Let $\mathcal{I}(G)$ be the family of all independent sets of $G$, and let $\mathcal{I}(G, v)$ be the family of all those independent sets which contain the vertex $v$. For each independent set $I$, consider a nonnegative

\footnotetext{
* Supported in part by the NSERC Discovery Grant R611450 (Canada), by the Canada Research Chairs program, and by the Research Project J1-8130 of ARRS (Slovenia).

${ }^{\dagger}$ Part of this work was done while the author was a PIMS Postdoctoral Fellow at the Department of Mathematics, Simon Fraser University, Burnaby, B.C.
} 
real variable $y_{I}$. The fractional chromatic number of $G$, denoted by $\chi_{f}(G)$, is the minimum value of

$$
\sum_{I \in \mathcal{I}(G)} y_{I}, \quad \text { subject to } \sum_{I \in \mathcal{I}(G, v)} y_{I} \geq 1 \text { for each } v \in V(G) .
$$

Erdős actually proved in [6] that there exist graphs with large girth and large fractional chromatic number. In this paper, we prove the fractional chromatic number version of Rödl's result.

Theorem 3. For every real number $x \geq 1$, there exists a positive number $k(x)$ such that every graph $G$ with $\chi_{f}(G) \geq k(x)$ contains a triangle-free subgraph $H$ with $\chi_{f}(H) \geq x$.

Given this result, we put forward the following.

Conjecture 4. For every real number $x \geq 1$ and any integer $g \geq 3$, there exists a positive number $k(x, g)$ such that every graph $G$ with $\chi_{f}(G) \geq k(x, g)$ contains a subgraph $H$ of girth at least $g$ and with $\chi_{f}(H) \geq x$.

There is a natural question whether the chromatic number in the Erdös-Hajnal conjecture should be replaced by the fractional chromatic number. This leads us to graphs with large chromatic number and small fractional chromatic number. The most vulnerable examples in this respect might be Kneser graphs, for which the conjecture should be tested first. As our second contribution, we show in Section 3 that the Erdős-Hajnal Conjecture1 1 holds for Kneser graphs. The difference from the required value of the chromatic number of Kneser graphs versus the chromatic number of their subgraphs of large girth and chromatic number is very reasonable. See Theorem 16 and Corollary 17.

\section{Related problems}

The Erdős-Hajnal conjecture and the fact that random graphs provide examples of graphs of large girth and large fractional chromatic number motivates the following question. Let $G$ be any graph with large (fractional) chromatic number. Is it true that a random subgraph of $G$ will also have large (fractional) chromatic number (with high probability). Boris Bukh [3] asked a weaker question, whether a random subgraph of a graph with chromatic number $n$ has chromatic number comparably large to the chromatic number of a random graph of order $n$. More precisely, if $G$ is a graph and $p \in[0,1]$, we let $G_{p}$ denote a subgraph of $G$ where each edge of $G$ appears in $G_{p}$ independently with probability $p$.

Problem 1 (Bukh). Does there exist a constant $c$ so that for every graph $G$

$$
\mathbb{E}\left(\chi\left(G_{1 / 2}\right)\right)>c \frac{\chi(G)}{\log \chi(G)} ?
$$

The methods in this paper led us to a solution of the fractional version of Problem 1, see [11.

To put the Erdös-Hajnal conjecture in a bigger context, we cannot abide a related conjecture that was proposed in in 1983 by Carsten Thomassen.

Conjecture 5 (Thomassen [14]). For every positive integers $k$ and $g \geq 3$, there exists a positive number $d(k, g)$ such that every graph with average degree at least $d(k, g)$ contains a subgraph of girth at least $g$ and average degree at least $k$. 
Although this conjecture is still wide open, there are partial results. Kühn and Osthus [8] proved the conjecture for $g \leq 6$. A different proof of the same result is given in Dellamonica et al. [4]. Pyber et al. [12] and Dellamonica and Rödl [5] proved the conjecture under additional assumption when the maximum degree is not too large in terms of the average degree.

Here we propose a conjecture that is a weakening of Conjectures 1 and 5

Conjecture 6. For every positive integers $k$ and $g \geq 3$, there exists a positive number $c(k, g)$ such that every graph with chromatic number at least $c(k, g)$ contains a subgraph of girth at least $g$ and with average degree at least $k$.

While Thomassen's conjecture is trivial when we replace the girth condition by requesting that the odd girth is at least $g$ (meaning that the graph has no odd cycles of length less than $g$ ), the Erdős-Hajnal conjecture for odd girth may be of interest.

Conjecture 7. For every positive integers $k$ and $g$, there exists an integer $h(k, g)$ such that every graph $G$ with $\chi(G) \geq h(k, g)$ contains a subgraph with chromatic number at least $k$ and odd girth at least $g$.

\section{Proof of Theorem 3}

The proof of Theorem 3 uses the tools presented in this section.

Let $v_{1}, \ldots, v_{n}$ be a linear ordering of the vertices of a graph $G$, i.e. we consider $v_{i}<v_{j}$ whenever $i<j$. For a vertex $v$, let

$$
N^{L}(v)=\{u \mid u v \in E(G), u<v\},
$$

that is, the set of the neighbors of $v$ that appear before $v$. Rödl's proof of Theorem 2 is based on the following lemma (see [13]).

Lemma 8. If $\chi(G)>k^{t}$, and $\chi\left(N^{L}(v)\right) \leq t$ for every $v \in V(G)$, then there exists a triangle-free subgraph $H$ of $G$ with $\chi(H)>k$.

The original proof of Lemma 8 is elegant and very short, but it cannot be applied to the fractional chromatic number. Our major effort is to extend the above claim to the fractional chromatic number setup.

For a function $w: V(G) \rightarrow \mathbb{R}$, and a vertex-set $A$, we write $w(A)=\sum_{v \in A} w(v)$. The fractional independence number, denoted by $\alpha_{f}(G)$, is the minimum value of

$$
\max \{w(I) \mid I \in \mathcal{I}(G)\}
$$

where the minimum of (11) is taken over all non-negative weight functions $w$ with $w(V)=n$. By the linear programming duality we have the fact that $\chi_{f}(G)=\frac{n}{\alpha_{f}(G)}$, where $n=|V(G)|$. So we can consider our problem as a fractional independence number problem.

The following lemma is our statement on the fractional independence number analogous to Lemma 8. It uses the following function

$$
f(x, l)=x\left(\frac{\Gamma\left(x l^{7}+1\right)}{\Gamma(x+1)}\right)^{3}
$$


defined for real numbers $x \geq 1$ and $l \geq 1$, where $\Gamma(\cdot)$ stands for the Euler gamma function. We also fix a weight function $w: V(G) \rightarrow \mathbb{R}^{+}$on the vertices of $G$, with the assumption that $w(V) \neq 0$. The weight function $w$ defines a linear order $<$ on $V(G)$ such that $u<v$ implies that $w(u) \geq w(v)$; that is, the vertices are ordered starting with those with largest weight. In the proof of Theorem 3. the function $w$ will be the one minimizing (11) in the definition of the fractional independence number of $G$.

Lemma 9. Let $x \geq 59$ and $l \geq 1$. Suppose that $w(I) \leq \frac{w(V)}{f(x, l)}$ for every $I \in \mathcal{I}(G)$, and that $\chi_{f}\left(N^{L}(v)\right) \leq l$ for every $v \in V(G)$. Then $G$ contains a triangle-free spanning subgraph $H$, such that $w(I) \leq \frac{w(V)}{x}$ for every $I \in \mathcal{I}(H)$. In particular, $\chi_{f}(H) \geq x$.

Proof. If $1 \leq l<2$, then $G$ is triangle-free. Namely, if $G$ would contain a triangle uvz with $u<v<z$, then $u v$ would be an edge in $N^{L}(z)$, contradicting the assumption that $\chi_{f}\left(N^{L}(z)\right) \leq$ $l<2$. Moreover, the condition that $w(I) \leq w(V) / f(x, l)$ for every $I \in \mathcal{I}(G)$ implies that $\chi_{f}(G) \geq$ $f(x, l) \geq f(x, 1)=x$. Thus we can take $H=G$ if $1 \leq l<2$.

For $l \geq 2$, Lemma 9 is proved by induction together with Lemma 10. The latter one uses Lemma 9 in its proof with $l^{\prime}=l\left(1-\frac{1}{6(x+1)}\right)$ playing the role of $l$ and $x^{\prime}=x+1$ playing the role of $x$. Then Lemma 9 is proved for $l$ and $x$ by applying the other lemma. In order to see that this inductive proof scheme works, we need to argue that by applying the transformation from $(l, x)$ to $\left(l^{\prime}, x+1\right)$ consecutively a finite number of times, we eventually obtain the value $l^{\prime}$ which is between 1 and 2. To see this, let $l_{0}=l$ and for $i \geq 1$, let $l_{i}=l_{i-1}\left(1-\frac{1}{6(x+i)}\right)$ be the value of $l^{\prime}$ after $i$ steps, $i=1,2, \ldots$. The process stops if $l_{i}$ becomes smaller than 2. Observe that whenever this happens, $l_{i-1} \geq 2$ and thus $l_{i} \geq 2\left(1-\frac{1}{6(x+1)}\right)>1$. Moreover, note that $l_{i} \leq l_{i-1}-\frac{2}{6(x+i)}$ as long as $l_{i-1} \geq 2$. Thus,

$$
l_{i} \leq l_{0}-\frac{1}{3}\left(\frac{1}{x+1}+\frac{1}{x+2}+\cdots \frac{1}{x+i}\right) .
$$

Since the harmonic series diverges, the value of $l_{i}$ eventually becomes smaller than 2 . This justifies the inductive scheme of our proof. The rest of the proof of Lemma 9 is the inductive step proved for any $l \geq 2$; that part of the proof is given after the proof of Lemma 10.

Let $v_{1}, \ldots, v_{n}$ be the enumeration of the vertices in the non-increasing order according to the weight function $w$. Given a vertex-set $A$, let $A_{k}$ be the set of the first $k$ elements in $A$ according to this ordering. We extend this notion to all positive real numbers by setting $A_{s}:=A_{\lfloor s\rfloor}$.

For $s \in \mathbb{R}^{+}$, a nonempty subset $X$ of a vertex-set $Y$ is said to be $s$-principal in $Y$ if $X \subseteq Y_{s|X|}$. That is, if $X$ has size $m$, then all elements of $X$ are within the first $\lfloor s m\rfloor$ vertices in $Y$. On the other hand, a subset $X$ of $Y$ is $s$-sparse in $Y$ if $X$ contains no $s$-principal subset in $Y$. When the hosting set $Y$ for $s$-principal or $s$-sparse is not specified, by default it is the whole vertex-set $V$.

The next claim from [10] about the total weight of an $s$-sparse set will be essential for us. We include the proof for completeness.

Claim 1. Let $s>0$ be a real number. If $X$ is an s-sparse subset of $Y$, then $w(X) \leq \frac{1}{s} w(Y)$.

Proof. Let $y_{1}, \ldots, y_{r}$ be the non-decreasing order of the elements of $Y$ with $r=|Y|$, and let $x_{1}, \ldots, x_{m}$ be the ordering of $X$ with $m=|X|$. Since $X$ is an $s$-sparse subset of $Y$, we have $x_{i} \notin Y_{s i}$. Hence for $1 \leq i \leq m, w\left(x_{i}\right) \leq w\left(y_{j}\right)$ if $1 \leq j \leq$ si. Moreover, since $x_{i} \in Y \backslash Y_{s i}$, we also have $w\left(x_{i}\right) \leq w\left(y_{j}\right)$ for $j=\lceil s i\rceil$. 
For a real parameter $z \in(0,|Y|]$, define $f(z)=y_{\lceil z\rceil}$. Then $f(z) \geq w\left(x_{1}\right)$ for $0<z \leq s$, $f(z) \geq w\left(x_{2}\right)$ for $s<z \leq 2 s, \ldots, f(z) \geq w\left(x_{m}\right)$ for $(m-1) s<z \leq m s$. Therefore,

$$
s w(X)=s \sum_{i=1}^{m} w\left(x_{i}\right) \leq \int_{0}^{s m} f(z) d z \leq \sum_{j=1}^{\lceil s m\rceil} w\left(y_{j}\right) \leq w(Y),
$$

which gives what we were aiming to prove.

Let $A \subseteq V$ be a vertex-set and $v \in A$. Let $L_{A}(v)$ be the subgraph of $G$ induced by the neighbors of $v$ in $A$ that appear before $v$. The set $A$ is $(x, l)$-reducible if it satisfies the following conditions:

(i) $w(A) \geq \frac{w(V)}{x(x+1)^{2}}$ and

(ii) $\chi_{f}\left(L_{A}(v)\right) \leq l\left(1-\frac{1}{6(x+1)}\right)$ for every $v \in A$.

Lemma 10. Suppose that $x \geq 3$ and $l \geq 2$ are real numbers. Suppose that Lemma 9 holds for $x^{\prime}=$ $x+1$ and $l^{\prime}=l-\frac{l}{6(x+1)}$. Suppose that every independent set I in $G$ satisfies $w(I) \leq w(V) / f(x, l)$. For any $(x, l)$-reducible set $A$, there is a triangle-free subgraph $H_{A}$ with $V\left(H_{A}\right)=A$ such that any independent set of $H_{A}$ has weight at most $\frac{w(A)}{x+1}$.

Proof. Note that $f(x, 1)=x$. Moreover, $f(x, l)$ satisfies the following recursive bound:

$$
f(x, l) \geq x(x+1)^{2} f\left(x^{\prime}, l^{\prime}\right) .
$$

A short calculation combined with the fact that the gamma function $\Gamma(t)$ is increasing for $t \geq 2$ shows that (2) holds if and only if $(x+1)\left(1-\frac{1}{6(x+1)}\right)^{7} \leq x$. By using elementary calculus, it is easy to verify that this inequality holds for every $x \geq 3$. This confirms (2).

For any independent set $I \subseteq A$, we have

$$
w(I) \leq \frac{w(V)}{f(x, l)} \leq \frac{w(A)}{\frac{f(x, l)}{x(x+1)^{2}}} \leq \frac{w(A)}{f\left(x^{\prime}, l^{\prime}\right)} .
$$

By our assumption, Lemma 9 holds for $x^{\prime}$ and $l^{\prime}$. Therefore, there is a triangle-free subgraph $H_{A}$ with $V\left(H_{A}\right)=A$ such that any independent set in $H_{A}$ has weight at most $\frac{w(A)}{x+1}$.

In what follows, we will prove Lemma 9 with the help of Lemma 10, By our inductive scheme described before, we may assume that Lemma 9 holds for $x^{\prime}$ and $l^{\prime}$, and thus the conclusion of Lemma 10 can be used.

Let $\mathcal{R}$ be a maximal collection of pairwise disjoint $(x, l)$-reducible sets and let $R=\cup \mathcal{R}$ be the union of all these sets. Then the complement $\bar{R}=V \backslash R$ contains no reducible subsets. Applying Lemma 10 for each $A \in \mathcal{R}$, we can find a triangle-free spanning subgraph $H_{A}$ of $G(A)$, such that for any independent set $I \subset V\left(H_{A}\right)=A$, we have $w(I) \leq \frac{w(A)}{x+1}$. We let $H_{0}=\cup_{A \in \mathcal{R}} H_{A}$. Then every $I \in \mathcal{I}\left(H_{0}\right)$ is disjoint union of independent sets in subgraphs $H_{A}(A \in \mathcal{R})$ and thus

$$
w(I) \leq \sum_{A \in \mathcal{R}} \frac{w(A)}{x+1}=\frac{w(R)}{x+1}
$$


Let $L_{G}(v)$ denote the graph $L_{V(G)}(v)$. By the assumption of Lemma 9, we have $t=t(v):=$ $\chi_{f}\left(L_{G}(v)\right) \leq l$. Let $\mathcal{I}(v)$ be the collection of independent sets of $L_{G}(v)$. There exists a weight function $u: \mathcal{I}(v) \rightarrow[0,1]$, such that any vertex in $L_{G}(v)$ is covered by independent sets with total weight at least 1 , and total weight of $\mathcal{I}(v)$ is $t$. For a set $A$ containing $v$, we say $v$ is type 1 in $A$ if the total $u$-weight of those sets in $\mathcal{I}(v)$ that are disjoint from $A$ is at most $\frac{t}{6(x+1)}$; otherwise $v$ is type 2 in $A$. Let $T_{1}(A)$ be the collection of type 1 vertices in $A$ and $T_{2}(A)$ be the collection of type 2 vertices in $A$.

A nonempty vertex-set $A \subseteq \bar{R}$ is said to be dense if $A$ is $(x+1)$-principal in $\bar{R}$ and $\left|T_{2}(A)\right| \leq \frac{|A|}{x+1}$.

Lemma 11. G contains a triangle-free spanning subgraph $H$ such that no dense subset of $\bar{R}$ is independent in $H$.

Proof. We start by taking the subgraph $H_{0}$ with vertex-set $R$ defined above. Then we define a subgraph $H$ by adding the vertices of $\bar{R}$ and some edges from these vertices to the rest of the graph by using the following random choice. For each vertex $v \in \bar{R}$, randomly pick an independent set $I$ from $\mathcal{I}(v)$ according to their weight $u$, and then add the edges between $I$ and $v$ to $H$. Now, the lemma follows from Claims 2 and 3 that are proved below.

Claim 2. $H$ is a triangle-free subgraph of $G$.

Proof. Suppose there is a triangle with vertices $v_{i}, v_{j}, v_{k}$. Since $H_{0}$ is triangle-free, not all of these vertices are in $R$. Let $v_{r} \in\left\{v_{i}, v_{j}, v_{k}\right\} \cap \bar{R}$ be the one with $r$ largest possible. Then the two edges from $v_{r}$ to the other two vertices in $\left\{v_{i}, v_{j}, v_{k}\right\}$ have been added by the random choice at $v_{r}$. However, $v_{r}$ was joined to an independent set, and thus the other two vertices cannot be adjacent. This is in contradiction to $v_{i} v_{j} v_{k}$ being a triangle in $H$.

Claim 3. The probability that every dense subset of $\bar{R}$ contains an edge in $H$ is positive.

Proof. For a dense subset $A \subseteq \bar{R}$ of cardinality $k$, at least $k-\frac{k}{x+1}=\frac{k x}{x+1}$ vertices of $A$ are type 1. For each type 1 vertex $v$ of $A$, the total weight of sets in $\mathcal{I}(v)$ is $t=t(v) \leq l$, and the total weight of those that are out of $A$ is at most $\frac{t}{6(x+1)}$. Therefore, the selected independent set $I$ from $\mathcal{I}(v)$ has no vertices in $A$ with probability at most $\frac{1}{6(x+1)}$. Therefore, the probability that $A$ is an independent set in $H$ is at most $\left(\frac{1}{6(x+1)}\right)^{\frac{k x}{x+1}}$.

As every dense subset of $\bar{R}$ is $(x+1)$-principal, there are at most $\left(\begin{array}{c}\lfloor k(x+1)\rfloor \\ k\end{array}\right)$ dense sets of order $k$. By Stirling's Formula, $k ! \geq \sqrt{2 \pi k}\left(\frac{k}{e}\right)^{k}>\left(\frac{k}{e}\right)^{k}$. Thus, we have

$$
\left(\begin{array}{c}
\lfloor k(x+1)\rfloor \\
k
\end{array}\right)<\frac{(k(x+1))^{k}}{k !}<\frac{(k(x+1))^{k}}{\left(\frac{k}{e}\right)^{k}}=e^{k}(x+1)^{k} .
$$

Therefore, the probability that some dense set of order $k$ is an independent set in $H$ is less than

$$
e^{k}(x+1)^{k}\left(\frac{1}{6(x+1)}\right)^{\frac{k x}{x+1}}=\left(e 6^{-\frac{x}{x+1}}(x+1)^{\frac{1}{x+1}}\right)^{k} .
$$

If $x \geq 59$, then $e \cdot 6^{-\frac{x}{x+1}}(x+1)^{\frac{1}{x+1}}<1 / 2$, and thus the right-hand side in (4) is less than $2^{-k}$. So we have the probability that some dense set is an independent set in $H$ is less than $\sum_{k \geq 1} 2^{-k}<1$. With positive probability, every dense subset of $\bar{R}$ contains an edge in $H$. 
Lemma 12. If a set $S \subseteq \bar{R}$ contains no dense subset, then

$$
w(S) \leq \frac{w(\bar{R})}{x+1}+\frac{w(V)}{x(x+1)} .
$$

Proof. Let $S=\left\{s_{1}, s_{2}, \ldots, s_{|S|}\right\}$, where the enumeration is consistent with the weight $w$, i.e. $w\left(s_{i}\right) \geq$ $w\left(s_{j}\right)$ whenever $i \leq j$. For $1 \leq k \leq|S|$, we have $S_{k}=\left\{s_{1}, \ldots, s_{k}\right\}$ is not dense. This means that either $S_{k}$ is not $(x+1)$-principal in $\bar{R}$, or $\left|T_{2}\left(S_{k}\right)\right|>\frac{\left|S_{k}\right|}{x+1}$ (and $S_{k}$ is $(x+1)$-principal).

Let $S^{\prime}=\left\{s_{k} \mid S_{k}\right.$ is not $(x+1)$-principal in $\left.\bar{R}\right\}$. By the definition of sparse sets, it is easy to see that $S^{\prime}$ is $(x+1)$-sparse in $\bar{R}$. By Claim 1, we have $w\left(S^{\prime}\right) \leq \frac{1}{x+1} w(\bar{R})$.

Let $S^{\prime \prime}=S \backslash S^{\prime}=\left\{s_{k} \mid S_{k}\right.$ is $(x+1)$-principal in $\left.\bar{R}\right\}$. We can enumerate the elements in $S^{\prime \prime}$ as $s_{i_{1}}, s_{i_{2}}, s_{i_{3}}, \ldots$, where $1 \leq i_{1}<i_{2}<i_{3}<\cdots$. If $S_{k}$ is $(x+1)$-principal in $\bar{R}$, since $S$ does not contain any dense set and by the definition of dense set, we have $\left|T_{2}(S) \cap S_{k}\right|=\left|T_{2}\left(S_{k}\right)\right|>\frac{\left|S_{k}\right|}{x+1}=\frac{k}{x+1}$. This implies that $T_{2}(S)$ contains elements $s_{r_{1}}, s_{r_{2}}, s_{r_{3}}, \ldots$, where $1 \leq r_{1}<r_{2}<r_{3}<\cdots$ such that $r_{1} \leq i_{1}, r_{2} \leq i_{\lceil x+1\rceil}, r_{3} \leq i_{\lceil 2(x+1)\rceil}$, etc. As a consequence we obtain the following:

$$
\begin{aligned}
(x+1) w\left(s_{r_{1}}\right) & \geq \sum_{r=1}^{x+1} w\left(s_{i_{r}}\right), \\
(x+1) w\left(s_{r_{2}}\right) & \geq \sum_{r=x+2}^{2(x+1)} w\left(s_{i_{r}}\right),
\end{aligned}
$$

etc. (The sums in these inequalities are to be understood in the same way as in the proof of Claim 1. where we used integration in order to be precise: If the value $r$ of the upper (or lower) bound in the summation is not an integer, then we add partial value of the corresponding weight $w\left(s_{i_{\lceil r\rceil}}\right)$ $\left(w\left(s_{i_{\lfloor r\rfloor}}\right)\right)$ proportional to the distance from the "floor" (the "ceiling") of the value.) By summing up these inequalities, we get $(x+1) w\left(T_{2}(S)\right) \geq w\left(S^{\prime \prime}\right)$.

As $T_{2}(S)$ is not reducible, we have one of the following outcomes: either $w\left(T_{2}(S)\right)<\frac{w(V)}{x(x+1)^{2}}$ or $\chi_{f}\left(L_{T_{2}(S)}(v)\right)>l\left(1-\frac{1}{6(x+1)}\right)$ for some $v \in T_{2}(S)$. We claim that the second outcome contradicts our assumptions. By the definition of $T_{2}(S)$, for each $v \in T_{2}(S)$, the total weight of independent sets in $\mathcal{I}(v)$ that are disjoint from $S$ is at least $\frac{t}{6(x+1)}$ (where $t=t(v) \leq l$ ), the other independent sets have total weight at most $t\left(1-\frac{1}{6(x+1)}\right)$. This corresponds to a fractional coloring of $L_{T_{2}(S)}(v)$ with order $t\left(1-\frac{1}{6(x+1)}\right) \leq l\left(1-\frac{1}{6(x+1)}\right)<\chi_{f}\left(L_{T_{2}(S)}(v)\right)$, which is a contradiction. So we must have $w\left(T_{2}(S)\right)<\frac{w(V)}{x(x+1)^{2}}$. Hence $w\left(S^{\prime \prime}\right) \leq(x+1) w\left(T_{2}(S)\right)<\frac{w(V)}{x(x+1)}$.

Now we have $w(S)=w\left(S^{\prime}\right)+w\left(S^{\prime \prime}\right)<\frac{w(\bar{R})}{x+1}+\frac{w(V)}{x(x+1)}$.

Let $I$ be an independent set in $H$. By Lemma 11, $I$ contains no dense subsets. By (3) and Lemma 12 we have

$$
\begin{aligned}
w(I) & =w(I \cap R)+w(I \cap \bar{R}) \\
& \leq \frac{w(R)}{x+1}+\frac{w(\bar{R})}{x+1}+\frac{w(V)}{x(x+1)} \\
& =w(V)\left(\frac{1}{x+1}+\frac{1}{x(x+1)}\right)=\frac{w(V)}{x}
\end{aligned}
$$


This completes the proof of Lemma 9.

We are ready to give the proof of Theorem 3 .

Proof of Theorem (3. Clearly, we may assume that $x \geq 59$ by setting $k(x)=k(59)$ for every $x<59$.

Let $r(x)$ be the smallest integer such that there exists a triangle-free graph $Q$ of order $r(x)$ with $\chi_{f}(Q) \geq x$. (It follows by the known bounds on Ramsey numbers $R(3, t)[9$ ] and a result by Ajtai, Komlós, and Szemerédi [1] that $r(x)=\Theta\left(x^{2} / \log x\right)$.) Define $k_{0}(x)=x$ and set $k_{t}(x)=f\left(x, k_{t-1}(x)\right)$ for $t=1,2, \ldots, r(x)$. Finally, let $k(x)=k_{r(x)}(x)$.

Let $t=r(x)$ and let $G_{t}=G$ be a graph with $\chi_{f}(G) \geq k(x)=k_{t}(x)$. Consider the weight function $w_{t}$ which minimizes (11) in the definition of the fractional independence number of $G_{t}$. If there is a vertex $v_{t}$ such that $\chi_{f}\left(N^{L}\left(v_{t}\right)\right)>k_{t-1}(x)$, then we consider the subgraph induced on $N^{L}\left(v_{t}\right)$ and decrease $t$ by 1 . This new graph defines the new weight function and for the decreased value of $t$ we repeat the same test whether there is a vertex $v_{t}$ such that $\chi_{f}\left(N^{L}\left(v_{t}\right)\right)>k_{t-1}(x)$. If we decrease $t$ all the way down to 1 , then the vertices $v_{1}, v_{2}, \ldots, v_{r(x)}$ form a complete subgraph of $G$, and by the definition of $r(x)$, there is a triangle-free subgraph isomorphic to $Q$, whose fractional chromatic number is at least $x$. This gives the desired outcome of the theorem.

On the other hand, if the process stops at $t>1$, we have obtained a graph $G_{t}$ such that $\chi_{f}\left(G_{t}\right) \geq k_{t}(x)=f\left(x, k_{t-1}(x)\right)$ and for every vertex $v$, we have $\chi_{f}\left(N^{L}\left(v_{t}\right)\right) \leq k_{t-1}(x)$. By Lemma 9. $G_{t}$ (and hence also $G$ ) contains a triangle-free subgraph $H$ with $\chi_{f}(H) \geq x$.

\section{Blow-ups and Kneser graphs}

The fact that the Erdős-Hajnal Conjecture is so resistant and the results of this paper open the question whether graphs with bounded fractional chromatic number (and large chromatic number) would still satisfy the conjecture. The most natural examples of such graphs are Kneser graphs. In this section we give the proof that the Erdös-Hajnal Conjecture holds for them.

Let us recall that the vertex-set of the Kneser graph $\operatorname{KG}(n, k)$ consists of all $k$-sets of elements of $\{1, \ldots, n\}$, and two such sets are adjacent if they are disjoint. It may be assumed that $k \leq n / 2$. It is known that $\chi(\mathrm{KG}(n, k))=n-2 k+2$ and that $\chi_{f}(\mathrm{KG}(n, k))=n / k$.

Given a graph $H$, the blow-up of $H$ with power $m$, denoted by $H^{(m)}$, is the graph obtained from $H$ by replacing each vertex by an independent set of size $m$ (called the blow-up of the vertex), and for each edge $x y$ in $H$, the two blow-ups of $x$ and $y$ form a complete bipartite graph $K_{m, m}$. The subgraph of $H^{(m)}$ replacing an edge $x y$ of $H$ is isomorphic to $K_{m, m}$ and will be referred to as the blow-up of that edge.

We have the following statement.

Theorem 13. Suppose $G$ is a graph with $\Delta(G) \leq \Delta$ and $\chi(G)>x$. Suppose that $m$ is an integer that is larger than $x(x \Delta)^{2 g-4}$. Then there exists a subgraph $H$ of $G^{(m)}$ with girth more than $g$ and chromatic number more than $x$.

There are a few existing papers, for example [2, 15], in which a result similar to Theorem[13] was proved. (The corresponding results in [2, 15] were applied to a construction of uniquely colorable graphs of large girth.) But the bound for the blow-up power $m$ in [2] and [15] is too large for our purpose as it depends on the number of vertices of $G$ instead of the maximum degree.

To prove Theorem [13, we will need the following fact from [2]. 
Lemma 14. Given a graph $G$ with $\chi(G)>x$, let $H$ be a subgraph of $G^{(m)}$. Suppose that for any edge $a b \in E(G)$ and for any subsets $X, Y$ contained in the blow-ups of a and $b$, respectively, with $|X| \geq \frac{m}{x},|Y| \geq \frac{m}{x}$, there is an edge between $X$ and $Y$ in $H$. Then $\chi(H)>x$.

In the proof of Theorem 13 we will take a random subgraph $H$ of $G^{(m)}$, obtained by selecting each edge independently with probability $\left(\frac{m}{x}\right)^{\frac{1}{4 l}-1}$, and will prove that with positive probability $H$ has no short cycles, and for any edge $a b \in E(G)$ and for any pair $X, Y$ contained in the respective blow-ups of $a$ and $b$, and with $|X| \geq \frac{m}{x},|Y| \geq \frac{m}{x}$, there is an edge between $X$ and $Y$ in $H$. To prove this, we will use the following asymmetric form of the Lovász Local Lemma.

Theorem 15 (Lovász Local Lemma). Let $\mathcal{A}=\left\{A_{1}, \ldots, A_{n}\right\}$ be a finite set of events in the probability space $\Omega$. For $A \in \mathcal{A}$ let $\Gamma(A)$ denote a subset of $\mathcal{A}$ such that $A$ is independent from the collection of events $\mathcal{A} \backslash(\{A\} \cup \Gamma(A))$. If there exists an assignment of real numbers $y: \mathcal{A} \rightarrow(0,1)$ to the events such that

$$
\forall A \in \mathcal{A}: \operatorname{Pr}(A) \leq y(A) \prod_{B \in \Gamma(A)}(1-y(B))
$$

then the probability of avoiding all events in $\mathcal{A}$ is positive. In particular,

$$
\operatorname{Pr}\left(\overline{A_{1}} \wedge \ldots \wedge \overline{A_{n}}\right) \geq \prod_{A \in \mathcal{A}}(1-y(A)) .
$$

Proof of Theorem 13. We can assume $x \neq 2$, since there is an odd cycle with length more than $g$ in $G^{(m)}$.

Let $s=\frac{m}{x}, \lambda=\frac{1}{4 g}$ and $p=s^{\lambda-1}$. Let $H$ be a random subgraph of $G^{(m)}$ obtained by picking each edge in $G^{(m)}$ independently with probability $p$.

If $C$ is a cycle in $G^{(m)}$ of length at most $g$, let $A_{C}$ be the event that all edges of $C$ appear in $H$. Then $\operatorname{Pr}\left(A_{C}\right)=p^{|C|}=s^{(\lambda-1)|C|}$.

Let $B$ be a copy of $K_{s, s}$ as a subgraph of a blow-up of an edge in $G$, let $A_{B}$ be the event that $H$ contains none of the edges of $B$. Then $\operatorname{Pr}\left(A_{B}\right)=(1-p)^{s^{2}} \approx e^{-p s^{2}}=e^{-s^{1+\lambda}}$.

To prove that there exists a subgraph of $G^{(m)}$ with girth at least $g$ and chromatic number at least $x$, we will use Lemma 14. We just need to show

$$
\operatorname{Pr}\left(\left(\bigwedge_{|C| \leq g} \overline{A_{C}}\right) \wedge\left(\bigwedge_{B \cong K_{s, s}} \overline{A_{B}}\right)\right)>0 .
$$

This will be confirmed by applying the asymmetric form of the Lovász Local Lemma (Theorem 15) to the two types of events together.

Suppose that $C$ is a cycle of length $|C| \leq g$. As the maximum degree in $G^{(m)}$ is at most $m \Delta=s x \Delta$, there are at most $|C|(s x \Delta)^{j-2}$ cycles of length $j$ in $G^{(m)}$ that share edges with $C$, and there are at most $|C|\left(\begin{array}{c}m \\ s\end{array}\right)^{2}$ copies of $K_{s, s}$ that share edges with $C$.

Suppose that $B$ is a copy of $K_{s, s}$. There are at most $s^{2}(s x \Delta)^{j-2}$ cycles of length $j$ in $G^{(m)}$ that share edges with $B$, and there are at most $\left(\begin{array}{c}m \\ s\end{array}\right)^{2}$ copies of $K_{s, s}$ that share edges with $B$.

For the Lovász Local Lemma, let $y_{0}=y\left(A_{B}\right)=e^{-0.5 s^{1+\lambda}} \approx \operatorname{Pr}\left(A_{B}\right)^{0.5}$, and for each cycle $C$ of length $|C| \leq g$, let $y_{|C|}=y\left(A_{C}\right)=\operatorname{Pr}\left(A_{C}\right)^{1-\lambda}=s^{-(1-\lambda)^{2}|C|}<s^{(2 \lambda-1)|C|}$. We just need to show:

(1) $\operatorname{Pr}\left(A_{C}\right) \leq y_{|C|}\left(\prod_{3 \leq j \leq g}\left(1-y_{j}\right)^{|C|(s x \Delta)^{j-2}}\right)\left(1-y_{0}\right)^{|C|\left(\begin{array}{c}m \\ s\end{array}\right)^{2}}$;

(2) $\operatorname{Pr}\left(A_{B}\right) \leq y_{0}\left(\prod_{3 \leq j \leq g}\left(1-y_{j}\right)^{s^{2}(s x \Delta)^{j-2}}\right)\left(1-y_{0}\right)^{\left(\begin{array}{c}m \\ s\end{array}\right)^{2}}$. 
Let us take the logarithm on each side of the above inequalities, and use the fact that $0.9 \log (1-$ $z$ ) $>-z$ (when $z$ is close to 0 as it appears to be in our case when $z=y_{0}$ or $y_{j}$ ). After simplifying, we see that it suffices to verify the following inequalities:

$$
\begin{aligned}
& 0.9 \lambda(1-\lambda) \log s \geq \sum_{3 \leq j \leq g} s^{(2 \lambda-1) j}(s x \Delta)^{j-2}+e^{-0.5 s^{1+\lambda}}\left(\begin{array}{c}
s x \\
s
\end{array}\right)^{2} . \\
& 0.4 s^{1+\lambda} \geq \sum_{3 \leq j \leq g} s^{(2 \lambda-1) j} s^{2}(s x \Delta)^{j-2}+e^{-0.5 s^{1+\lambda}}\left(\begin{array}{c}
s x \\
s
\end{array}\right)^{2} .
\end{aligned}
$$

In order to prove (4), we first observe that:

$$
\begin{aligned}
\sum_{3 \leq j \leq g} s^{(2 \lambda-1) j} s^{2}(s x \Delta)^{j-2} & =\sum_{3 \leq j \leq g} s^{2 \lambda j}(x \Delta)^{j-2} \\
& <1.1 s^{2 \lambda g}(x \Delta)^{g-2} \\
& =1.1 s^{0.5}(x \Delta)^{g-2}
\end{aligned}
$$

By the assumption of the theorem, $s>(x \Delta)^{2 g-4}$. Thus, $(x \Delta)^{g-2}<s^{0.5}$, and we have

$$
\sum_{3 \leq j \leq g} s^{(2 \lambda-1) j} s^{2}(s x \Delta)^{j-2}<1.1 s
$$

Similarly, we also have $\sum_{3 \leq j \leq g} s^{(2 \lambda-1) j}(s x \Delta)^{j-2}<1.1 s^{-1}$, which will be used to prove (3).

As $\left(\begin{array}{c}s x \\ s\end{array}\right)^{2}<(e x)^{2 s}$, we have

$$
e^{-0.5 s^{1+\lambda}}\left(\begin{array}{c}
s x \\
s
\end{array}\right)^{2}<\left(e^{-0.5 s^{\lambda}}(e x)^{2}\right)^{s}=\left(e^{-0.5 s^{\lambda}+2+2 \log x}\right)^{s} .
$$

As $s>(x \Delta)^{2 g-4}$, we have $s^{\lambda}>(x \Delta)^{\frac{2 g-4}{4 g}}>4(1+\log x)$, hence $e^{-0.5 s^{1+\lambda}}\left(\begin{array}{c}s x \\ s\end{array}\right)^{2}<1$.

As $0.9 \lambda(1-\lambda) \log s \geq 0.9(1-\lambda) \frac{2 g-4}{4 g} \log (x \Delta) \geq 1.1 s^{-1}+1$ and $0.4 s^{1+\lambda} \geq 1.1 s+1$, we conclude that both inequalities (3) and (4) are true.

In summary, if $m=s x \geq x(x \Delta)^{2 g-4}$, by the asymmetric form of the Lovász Local Lemma, the event that $H$ has no cycles of length at most $g$ and every $K_{s, s}$ as a subgraph of a blow-up of an edge has at least one edge, has positive probability. Hence, by Lemma 14, the corresponding subgraph $H$ of $G^{(m)}$ has chromatic number more than $x$.

The following result from [10, Theorem 3.3] shows that Kneser graphs contain blow-ups of smaller Kneser graphs with large power.

Theorem 16. Let $n, k, t$, and $x$ be nonnegative integers such that $0<k<n$ and $x<k t$. The Kneser graph $\mathrm{KG}(n t, k t-x)$ contains the blow-up of $\mathrm{KG}(n, k)$ with power $\left(\begin{array}{c}k(-1) \\ x\end{array}\right)$ as a subgraph. Furthermore, when $x<t$, it contains the blow-up of $\operatorname{KG}(n, k)$ with power $\left(\begin{array}{c}k t \\ x\end{array}\right)$, and when $x=t$, it contains the blow-up of $\operatorname{KG}(n, k)$ with power $\left(\begin{array}{c}k t \\ x\end{array}\right)-k$.

From Theorem 13, we know that graphs that are blow-ups of smaller graphs with sufficiently large power satisfy the Erdős-Hajnal Conjecture. In particular, Kneser graphs are such examples. This can be used to derive the main result of this section. 
Corollary 17. The Erdös-Hajnal Conjecture holds for Kneser graphs.

Proof. Let $k$ and $g$ be the parameters from the Erdős-Hajnal Conjecture. Let $\mathrm{KG}(2 n, n-2 x)$ be a Kneser graph with large chromatic number. Since $\chi(\operatorname{KG}(2 n, n-2 x))=4 x+2$, this just means that $x$ is large in terms of $k$ and $g$. Let $t=x / k$. By Theorem 16, $\operatorname{KG}(2 n, n-2 x)$ contains a blow-up of $\operatorname{KG}(2 n / t,(n-x) / t)$ with power $m=\left(\begin{array}{c}(n-x)(t-1) / t \\ x\end{array}\right)$. (Note that $\operatorname{KG}(r, s)$ contains $\operatorname{KG}(r-1, s)$ as a subgraph, and thus, with some neglect of technicalities, we may assume that $x / k, 2 n / t,(n-x) / t$, etc. are integers.) Each vertex of $\mathrm{KG}(2 n / t,(n-x) / t)$ has degree $\left(\begin{array}{c}(n+x) / t \\ (n-x) / t\end{array}\right)=\left(\begin{array}{c}(n+x) / t \\ 2 x / t\end{array}\right)$, which is at most

$$
\Delta:=((n+x) / t)^{2 x / t}=\left(\frac{n k}{x}+k\right)^{2 k} .
$$

In order to apply Theorem 13, we need power $m \geq k(k \Delta)^{2 g-4}$ of a graph with chromatic number at least $k$. In the following we assume $x$ is large in terms of $g$ and $k$. Let us first consider the case when $n>x /\left(\frac{1}{2}-\frac{1}{2 k}\right)$. If we write $\frac{n}{x}=2+2 z$, this condition implies that $z \geq \frac{1}{k}$. If $x>k^{2}$, then

$$
\frac{n-x}{x} \cdot \frac{t-1}{t} \geq 1+z
$$

and

$$
\frac{n}{x}+1=3+2 z<(1+z)^{x / 2} .
$$

Suppose, moreover, that $x \geq 10 g k^{2} \log k$. Then

$$
k^{2 g(2 k+1)}<(1+z)^{x / 2} .
$$

Using inequalities (5) -(7), we obtain:

$$
\begin{aligned}
k(k \Delta)^{2 g-4} & =k^{2 g-3}\left(\frac{n k}{x}+k\right)^{2 k(2 g-4)} \\
& <\left(\frac{(n-x)(t-1) / t}{x}\right)^{x} \\
& <\left(\begin{array}{c}
(n-x)(t-1) / t \\
x
\end{array}\right)=m .
\end{aligned}
$$

On the other hand, when $x$ is large enough and $n \leq x /\left(\frac{1}{2}-\frac{1}{2 k}\right)$, then $n-2 x \leq n / k$ and $\mathrm{KG}(2 n, n-2 x)$ contains a blow-up of $\mathrm{KG}(k, 1)=K_{k}$ with large power.

Thus, we can apply Theorem 13 and conclude that $\operatorname{KG}(2 n, n-2 x)$ contains a subgraph with girth more than $g$ and chromatic number at least $k$.

\section{References}

[1] M. Ajtai, J. Komlós, E. Szemerédi, A note on Ramsey numbers, J. Combin. Theory Ser. A 29 (1980) 354-360.

[2] B. Bollobás, N. Sauer, Uniquely colourable graphs with large girth, Canad. J. Math 28 (1976), $1340-1344$. 
[3] Boris Bukh, Interesting problems, http://www.borisbukh.org/problems.html. See also http://www.openproblemgarden.org/category/bukh_boris

[4] D. Dellamonica, V. Koubek, D. M. Martin, V. Rödl, On a conjecture of Thomassen concerning subgraphs of large girth, J. Graph Theory 67 (2011), 316-331.

[5] D. Dellamonica, V. Rödl, A note on Thomassen's conjecture, J. Combin. Theory Ser. B 101 (2011), 509-515.

[6] P. Erdős, Graph theory and probability, Canad. J. Math. 11(1) (1959), 34-38.

[7] P. Erdős, Problems and results in combinatorial analysis and graph theory, in "Proof Techniques in Graph Theory" (ed. F. Harary), Academic Press, New York, 1969, pp. 27-35.

[8] D. Kühn, D. Osthus, Every graph of sufficiently large average degree contains a $C_{4}$-free subgraph of large average degree, Combinatorica 24 (2004), 155-162.

[9] J. H. Kim, The Ramsey number $\mathrm{R}(3, t)$ has order of magnitude $t^{2} / \log t$, Random Struct. Algorithms 7 (1995), 173-207.

[10] Bojan Mohar, Hehui Wu, Dichromatic number and fractional chromatic number, Forum of Mathematics, Sigma 4 (2016) e32, 14 pages. https://doi.org/10.1017/fms.2016.28

[11] Bojan Mohar, Hehui Wu, Fractional chromatic number of a random subgraph, submitted.

[12] L. Pyber, V. Rödl, E. Szemerédi, Dense graphs without 3-regular subgraphs, J. Combin. Theory Ser. B 63 (1995), 41-54.

[13] V. Rödl, On the chromatic number of subgraphs of a given graph, Proc. Amer. Math. Soc. 64 (1977), 370-371.

[14] Carsten Thomassen, Girth in graphs, J. Combin. Theory Ser. B 35 (1983), 129-141.

[15] Xuding Zhu, Uniquely $H$-colorable graphs with large girth, J. Graph Theory 23 (1996), 33-41. 